\title{
Our Surgical Results for Femoral Neck Fractures: A Demographic and Retrospective Cohort Study
}

\author{
Ertuğrul Allahverdi ${ }^{1}$ \\ ${ }^{1}$ Department of Orthopedics and Traumatology, Faculty of Medicine, Kafkas University, Kars, Turkey \\ Received: 11 March 2020, Accepted: 21 August 2020, Published online: 31 August 2020 \\ (C) Ordu University Institute of Health Sciences, Turkey, 2020
}

\begin{abstract}
Objective: Proximal Femur Fractures due to trauma in the form of falls have become a common health problem among advanced age groups worldwide.Proximal Femur Fractures occur in various anatomical regions due to low- and high-energy causes related to falls, traffic accidents, metastatic tumors and osteoporosis.

Method: We compared the WOMAC results in addition to post-operative ROMs between three groups covering healthy hip joints with no apparent pathology and operated hip joints in 34 cases.

Results: A total of 34 patients consisting of 19 males and 15 females were included in the study. The mean age was $62.91 \pm 5.13$ years in the proximal femur fracture was due to a traffic accident in 3 patients and a fall in 31 patients. Osteoporosis was present in 21 patients and osteopenia in 6 patients, with no bone pathology in 4 patients. Mild postoperative limitations were present in abduction and adduction on the operated joint side but there was no significant difference ( $p>0.05$ ) between the other ROM values of the operated side and the healthy side

Conclusion: The quality of life increased in the post-operative period according to the surgical method used, the selection of the appropriate materials for the reconstructive surgery performed, and early diagnosis and treatment.
\end{abstract}

Key words: Trauma, osteoporosis, fracture, femoral neck

Suggested Citation: Allahverdi E. Our Surgical Results for Femoral Neck Fractures: A Demographic and Retrospective Cohort Study. Middle Black Sea Journal of Health Science, 2020; 6(2):158-165.

\section{Address for correspondence/reprints:}

Ertuğrul Allahverdi

Telephone number: +90 (506) 4280784

ORCID-ID 0000-0001-7723-7338

E-mail: ertugrulallahverdi@ hotmail.com

DOI: $10.19127 / \mathrm{mbsjohs.702186}$

\section{Introduction}

Proximal Femur Fractures due to trauma in the form of falls have become a common health problem among advanced age groups worldwide. The increase in the prevalence of osteoporosis and the population over the age of 65 increases the incidence of femoral neck fractures. Hip fracture has the highest morbidity and mortality rates among all osteoporotic fractures. Approximately $50 \%$ of hip fracture patients suffer permanent postoperative functional limitation compared to their pre-fracture state. The incidence of hip fractures is increasing in many countries, especially in Asian developing countries, and the total number of hip fractures is predicted to reach more 
than five million by 2050 . While $26 \%$ of all hip fractures occurred in Asia in 1990, this rate is predicted to reach $37 \%$ in 2025 and $45 \%$ in 2050 . Femoral neck fractures will increase more significantly in males (310\%) than in females (240\%) (Gullberg et al., 1997). The socioeconomic effects of hip fracture can be considered in two ways: hip fractures increase morbidity and mortality in the elderly, and they increase health expenditure greatly in economic terms. There is therefore an urgent need to accurately evaluate the risk of hip fractures starting from the perspective of family medicine and then developing preventive and protective measures. Femoral neck fractures in the young age group are caused by trauma with exposure to high levels of energy. Various types of femoral neck fractures may be associated with multiple trauma. Early and accurate diagnosis is the most important criterion in the treatment of trauma patients. The risk of femoral head avascular necrosis and nonunion increases significantly with late diagnosis and treatment (Thuan and Swiontkowski, 2008; Butt et al., 2017; Patterson et al., 2018). Proximal Femur Fractures have been classified in various ways such as 1) as intra-capsular, extra-capsular (basal, trochanteric, subtrochanteric), 2) subcapital, medial, lateral, intertrochanteric, pertrochanteric, 3) according to the degree of angulation of the fracture line with the horizontal plane (Pauwels), 4) non-displaced due to fracture (Garden Type 1-2), displaced due to fracture (Garden Type 2-3), and 5) AO classification; subcapital (Type B1), transcervical (Tip B2), and displaced subcapital (Type B3). We performed surgical procedures according to classification 1 and 2 in our patients. Options in proximal femur fractures include Single or Double Lag Screw Proximal Femur Nail (PFN), 6.5 mm Cannulated Screw, Partial or Total Hip Prosthesis, and Dynamic Hip Screw (DHS) according to the type of the fracture, patient age, and bone density values (Gullberg et al., 1997; Malik et al., 2009; Li and Cole, 2015; Lin et al., 2015; Butt et al., 2017; Patterson et al., 2018).

\section{Methods}

Permission was obtained to conduct the study from the Kafkas University Ethics Committee with decision no 80576354-050-99/177.

Proximal femur fractures were detected in 34 patients between 2015 and 2019 according to the radiological and clinical examinations performed after referral to the Emergency Service by family physicians or other health institutions. Intracapsular and extracapsular fracture patients mostly underwent single and double lag screw proximal femur intramedullary nail implantation while cannulated triple screw implantation was used for the young patients with subcapital fractures, and partial hip prosthesis implantation for patients with extracapsular femoral neck fractures due to their advanced age (Figure 1,2,3,4). Group I consisted of patients who were implanted a single lag screw (PFNA) and cannulated screw $(\mathrm{n}=11)$, Group II consisted of patients who were implanted a double lag screw $(\mathrm{n}=10)$, and Group III consisted of patients with partial hip replacement $(n=13)$. In addition to the type of fracture, and according to the bone density quality, single lag screws and three cannulated screws were used in intracapsular fractures in the younger patients, double lag screws were used to provide better stability in those with intracapsular and extracapsular fractures, and partial hip prosthesis implantations were used in those with osteoporosis due to advanced age since the fracture improvement rate is low in elderly patients. Only patients with ROMs close to full on the healthy side hip joint and who had not undergone any surgery were included in our study. The movement capacities of the healthy and operated hip joints of our patients were measured with a Goniometer and recorded at the postoperative 3rd month. Besides, the WOMAC scores were determined and recorded at the postoperative 3rd month in all groups.
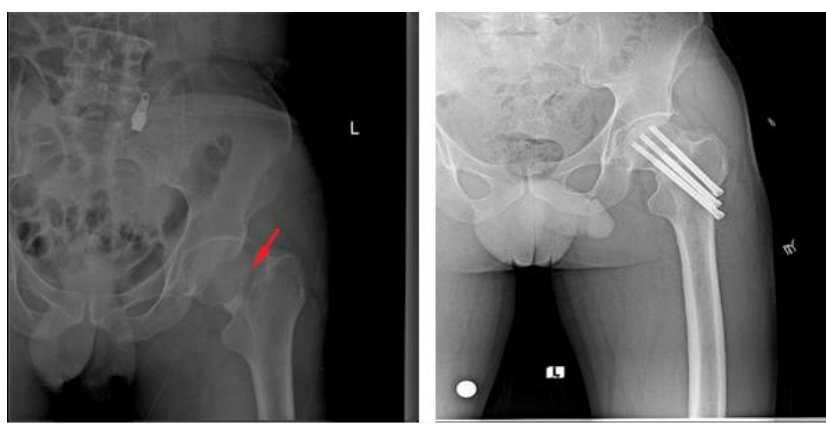

Figure 1. 37-year-old patient. Left intracapsular femoral neck fracture due to a traffic accident. Inverted triangular osteosynthesis with three $6.5 \mathrm{~mm}$ cannulated screws. 

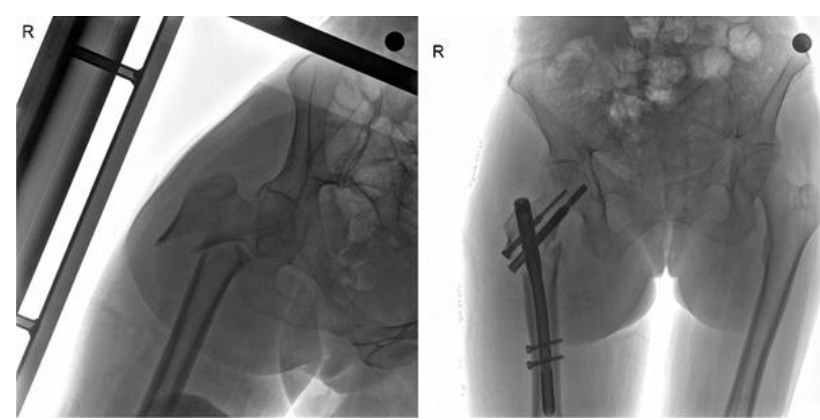

Figure 2. 55-year-old patient. Right extracapsular femoral neck fracture due to a traffic accident. Double Lag Screw PFN Implantation.

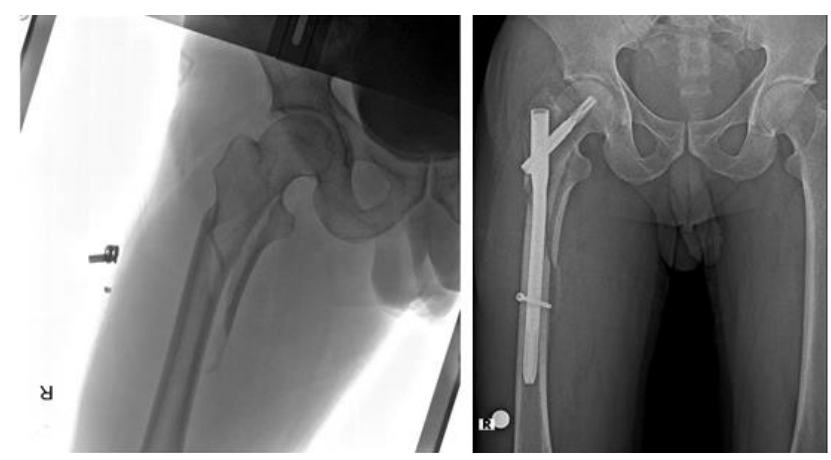

Figure 3. 49-year-old patient. Right extracapsular Rivers type femoral neck fracture. Single Lag Screw PFN Implantation
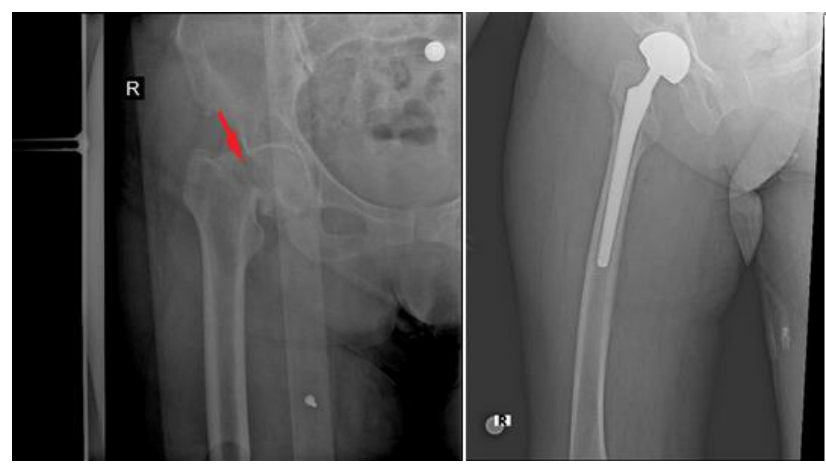

Figure 4. 93-year-old patient. Right intracapsular femoral neck fracture. Partial hip prosthesis implantation.

\section{Statistical analysis}

The normality test was applied to determine whether the data fit a normal distribution in the comparisons between the subgroups (Group I, II, III) for all parameters that were measured as regards the movement capacity. Since our data did not show a homogeneous distribution, Kruskal-Wallis analysis, which is one of the nonparametric tests, was applied to determine the difference between the groups for the measured parameters. Phenotypical correlation analysis was performed to determine the level of relationship between the parameters and WOMAC values in the subgroups (Group I, II, III) for all the measured parameters as related to movement capacity. The SPSS 20 software program was used for the descriptive statistics with the Kruskal-Wallis test while the Minitab 12 statistics software program was used for Phenotypical Correlation Analysis. The operated and non-operated extension values were removed in the correlation analysis because they were very close or the same between the patients. There was no significant difference between the groups in the Kruskal-Wallis analysis. The mean and standard error values of the groups are presented in Table 2 .

\section{Results}

A total of 34 patients consisting of 19 males and 15 females were included in the study. The mean age was $62.91 \pm 5.13$ years in Group I, $72.70 \pm 5.29$ years in Group II, and $82.92 \pm 1.70$ years in Group III. The proximal femur fracture was due to a traffic accident in 3 patients and a fall in 31 patients. Osteoporosis was present in 21 patients and osteopenia in 6 patients, with no bone pathology in 4 patients. Diabetes was present in 6 patients, Parkinson's disease in 2 patients, dementia in 5 patients, hypertension in 9 patients, chronic obstructive pulmonary disease (COPD) in 4 patients, nephropathy in 2 patients, goiter in 5 patients, smoking addiction in 17 patients, and alcohol abuse in 1 patient. The proximal femur fractures were on the right side in 17 patients and the left side in 17 patients. Peritrochanteric and reverse type proximal femur fractures were found in 14 patients, the lateral type in 8 patients, medial type in 6 patients, intertrochanteric type in 4 patients and subcapital type in 2 patients.

The parameters investigated in all the patient groups included Flexion, Extension, Internal Rotation, External Rotation, Abduction and Adduction capacity in both the healthy and operated hip joints. Whether the values were significant is summarized in Table 1 according to the correlation coefficients obtained by the comparison of these parameters. There was no significant difference in Flexion, Extension, Internal Rotation, or External Rotation between the healthy hip and operated hip joint. The operated hip joint capacity was close to that of the healthy hip, regardless of the group. The quality of life had not decreased due to the fracture at the post-operative $3 \mathrm{rd}$ month. A significant difference ( $\mathrm{p}$ $<0.001$ ) was found between the movement capacity of the operated hips. There was minor limitation in postoperative abduction and adduction in the operated side compared to the healthy side. The hip joint correlation showed significant values for the healthy side hip $(\mathrm{p}<0.01, \mathrm{p}<0.05)$ but not for the operated hip ( $p>0.05)$. The mean WOMAC (Western Ontario and McMaster Universities Osteoarthritis 
Index) (scoring of joint-related functions in terms of pain, stiffness and physical functions) values were determined in all groups at the postoperative 3rd month. A higher WOMAC score indicates worse post-operative patient satisfaction. No statistically significant difference was found between the WOMAC scores of our groups; however, there was a numerical difference between Group I -II and Group III. Accordingly, our patients in Group I-II had better post-operative joint movement and physical activity scores, and lower pain and stiffness analysis scores. These data are presented in Table 2. A significant $(p<0.05)$ correlation was found between the WOMAC and Age values. The disorders accompanying the proximal femoral fractures in all groups were also determined and are presented as percentages in Table 3, Table 4 presents the demographic features, fracture development mechanisms and the femoral neck fracture type of our patients.

Table 1. Correlation values (upper line) between the operated and non-operated hip movement capacities together with WOMAC values and the significance levels (lower line)

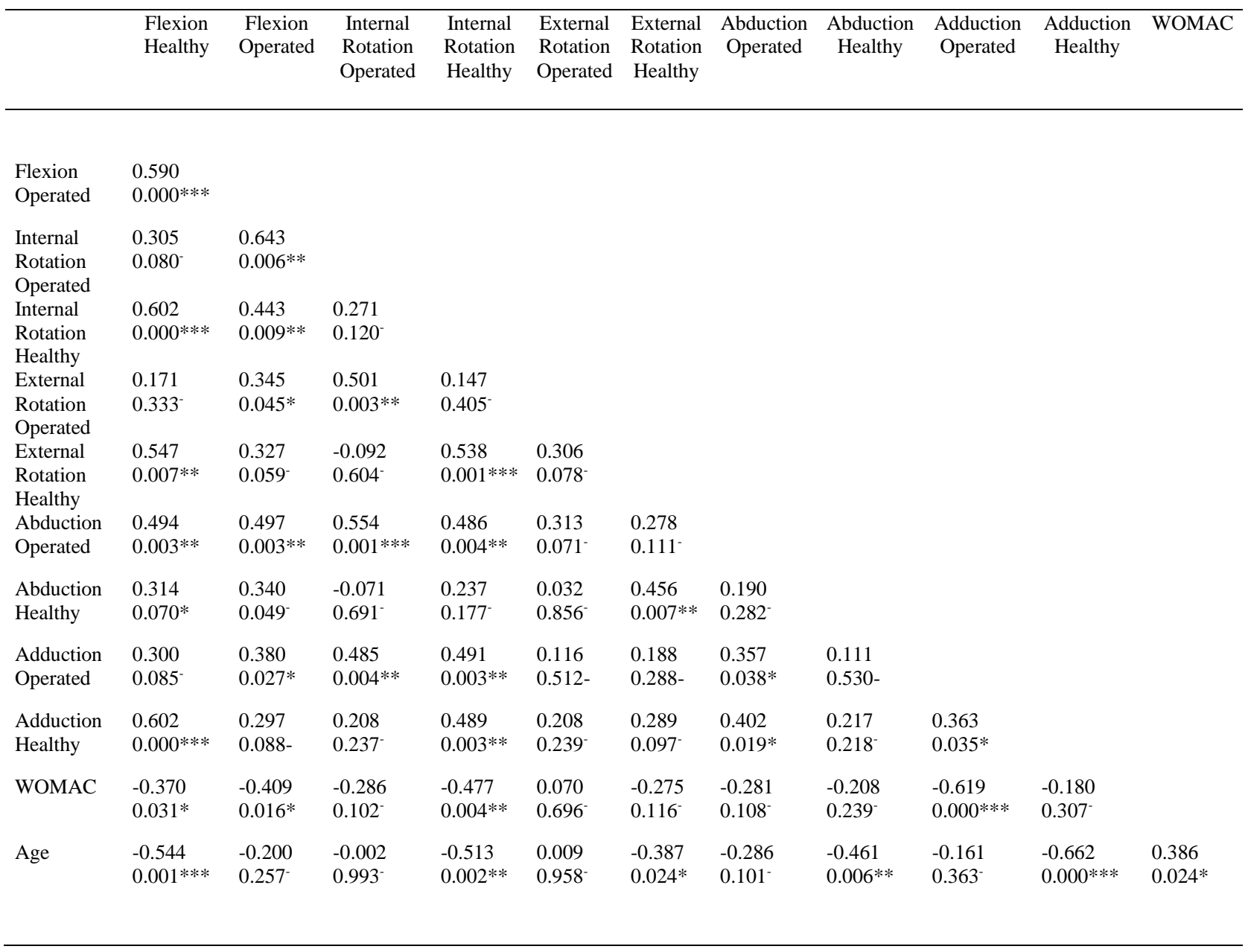

(*): $\mathrm{P}<0.05 ;(* *): \mathrm{P}<0.01 ;(* * *): \mathrm{P}<0.001 ;(-) ; \mathrm{P}>0.05$ Insignificant 


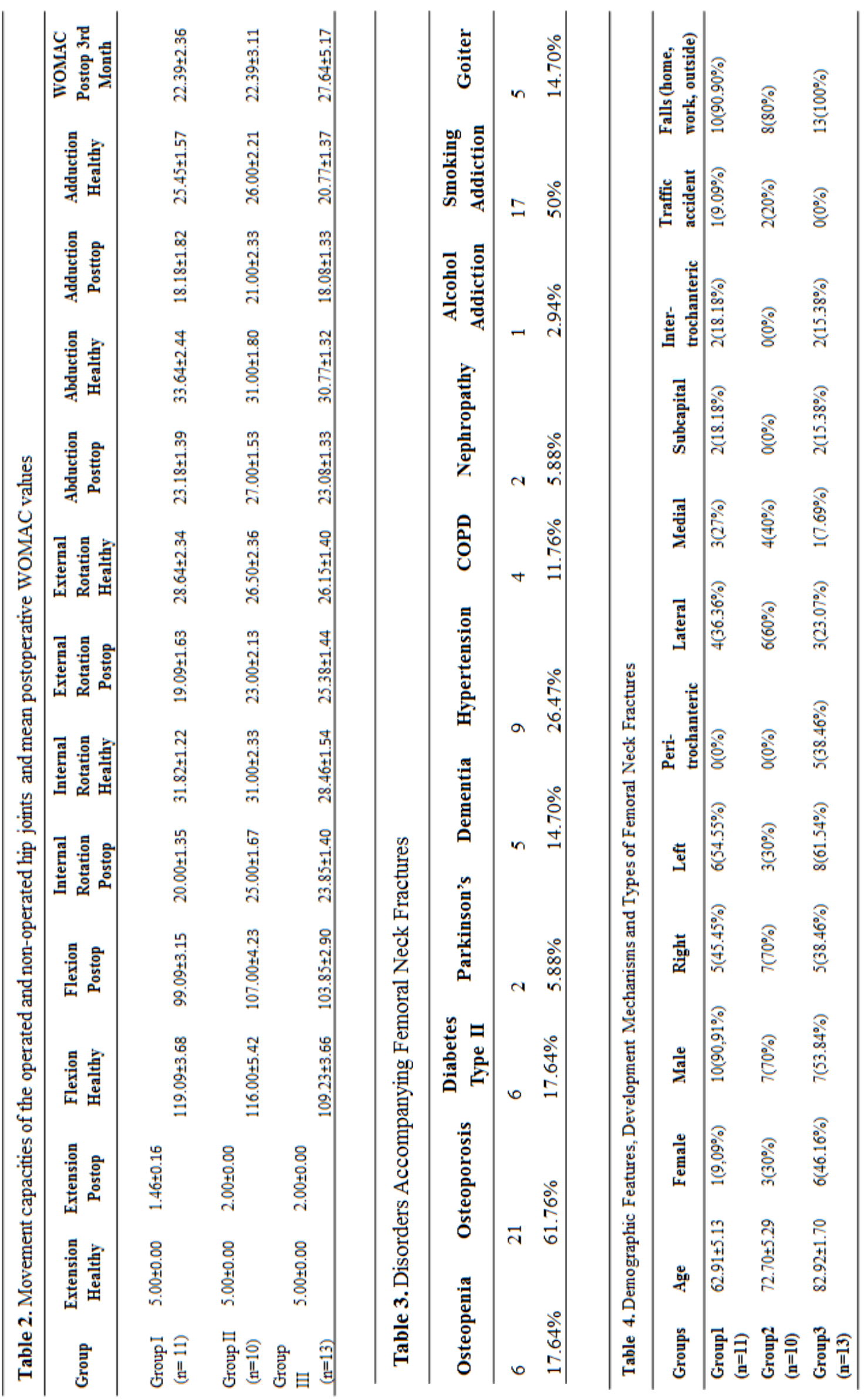




\section{Discussion}

Proximal Femur Fractures, which are commonly encountered in the elderly population, are usually pathological fractures due to osteoporosis. Proximal femur fractures in the young population are highenergy fractures and a serious health problem resulting in high nonunion and avascular necrosis (AVN) rates (Gullberg et al., 1997; Thuan and Swiontkowski, 2008; Malik et al., 2009; Li and Cole, 2015; Lin et al., 2015; Butt et al., 2017; Patterson et al., 2018). These fractures may occur due to Parkinson's disease, Alzheimer's disease, dementia, epilepsy, diabetic neuropathy, endocrine-metabolic diseases and in patients with alcohol or tobacco use, due to sprains and falls (Martyn, 2003; Thuan and Swiontkowski, 2008; Weil et al., 2015; Yamauchi et al., 2018). In our patients, osteoporosis was present in 21 patients, osteopenia in 6 patients, diabetes in 6 patients, Parkinson's disease in 2 patients, dementia in 5 patients, hypertension in 9 patients, chronic obstructive pulmonary disease (COPD) in 4 patients, nephropathy in 2 patients, goiter in 5 patients, smoking addiction in 17 patients, and alcohol abuse in 1 patient.

Proximal Femur Fractures require urgent and onsite intervention both in the younger age group (age $<60$ years) and in the elderly. Some authors have reported that intervention in the first 6-8 hours is required to ensure that the blood supply of the femoral neck is not impaired or that the impaired blood supply is restored. The AVN and nonunion rates are quite high in femoral neck fractures after late intervention (Martyn, 2003; Thuan and Swiontkowski, 2008; Yamauchi et al., 2018). Both young and elderly patients with hip pain should therefore be evaluated clinically and radiologically.

The gold standard in the treatment of femoral neck fractures consists of early diagnosis, using the appropriate surgical procedure, anatomical reduction, intraoperative capsular decompression and stable fixation for a cannulated screw or the PFN and DHS procedures. Early diagnosis and treatment are two important factors to ensure fracture healing without any complications. The patient with a proximal femur fracture should therefore be diagnosed at the time of the patient encounter so that treatment can be arranged (Braun et al., 1991; Parker, 2000; Ong et al., 2002; Bryant and Harley, 2007; Langford and Strauss, 2008; Lee et al., 2008; Thuan and Swiontkowski, 2008; Zlowodzki et al., 2008; Florschutz et al., 2015; Weil et al., 2015). All of our cases were evaluated at the emergency service and underwent surgery as soon as possible after a diagnosis was made clinically and radiologically.
Postoperative avascular necrosis did not develop in 21 of our 34 patients thanks to our femoral neck and head protective surgical methods. There was also no infection in any of our patients. Deep vein thrombosis and concomitant pulmonary embolism were found in three patients in the third and fourth postoperative weeks.

Varization was found after PFN implantation in one patient. Revision was planned for this patient but could not be performed due to advanced cardiopulmonary disease. The patient continued his daily life using a single cane.

No prosthetic loosening, luxation, new fracture or implant failure was observed in any of the 34 patients in the postoperative period. Passive and active mobilization was started postoperatively in all of our patients. Some studies have reported permanent postoperative functional limitation compared to the pre-fracture values in approximately $50 \%$ of the patients (Gullberg et al., 1997). Post-operative hip movement was close to that of the intact hip after recovery. Two of our patients could only start active mobilization later due to advanced stage Parkinson's disease and dementia. Calcium and Vitamin D3 were regularly started in all of our patients regardless of age. A minimum of 3 months is recommended to support the remodeling period of the fracture and to support porous healing of prostheses in cases of widespread regional vitamin $\mathrm{D}$ deficiency. Bisphosphonate derivatives were added to the treatment of the patients diagnosed with osteoporosis who had not received osteoporosis treatment or regular medical care. We found that WOMAC scores were lower in Group I and II compared to Group III with older age but there was no statistically significant difference. Our data are also supported by the literature (Braun et al., 1991; Thuan and Swiontkowski, 2008; Florschutz et al., 2015).

Multiple factors such as the femoral neck fracture type, patient age, presence of additional diseases, time and type of patient presentation, the bone quality of the patient, and patient habits and addictions that can negatively affect nutrition and healing by influencing the vascularity in the fractured bones all play a role (Gullberg et al., 1997; Thuan and Swiontkowski, 2008; Florschutz et al., 2015; Lin et al., 2015; Weil et al., 2015). Our cases in Group I and II underwent post-traumatic surgical stabilization within 4 to 48 hours. Partial hip prosthesis implantation was performed within 24 to 72 hours in Group III. The bone quality was better in Group I and II than in Group III. The joint movement ROMs were lower and the WOMAC scores were higher in Group III, despite the lack of statistical significance, due to 
more significant comorbidity but with no difference regarding movement capacity and mobilization. Our patients in Group I-II had better post-operative joint movement and physical activity scores, and lower pain and stiffness analysis scores.

There was no infection in any of our patients. Deep vein thrombosis and concomitant pulmonary embolism were found in three patients in the third and fourth postoperative weeks. Postoperative avascular necrosis did not develop of our patients. This may also have decreased the socio-economic costs of femoral neck fracture.

\section{Conclusion}

Early diagnosis and treatment, correct selection of the surgical procedures and materials to be used according to the indications, the control of comorbidity with a multidisciplinary approach, early surgical intervention, post-operative initiation of medical treatment for osteoporosis, and early mobilization increase tolerability in the early period and decrease the revision surgery rate. We recommend studies on a larger number of patients and with a longer follow-up period according to different and detailed new indexes for better analysis of our correlations with higher reliability.

Ethics Committee Approval: The study was approved by the ethics committee of Kafkas University (Decision No: 80576354-050-99/177). The study was performed following the aid of the ethical standards down in the 1964 Declaration of Helsinki and its later amendments.

Peer-review: Externally peer-reviewed.

Author Contributions: Concept- E.A.; DesignE.A.; Materials- E.A.; Data Collection and Processing- E.A.; Literature Review- E.A.; WritingE.A.; Critical Review- E.A.

Conflict of Interest: No conflict of interest was declared by the author.

Financial Disclosure: The author declared that this study hasn't received no financial support.

\section{References}

Braun W, Rüter A, Wiedemann M, Kissing F. Femoral head preserving therapy in medial femoral neck fractures. A clinical study of the effects of treatment method on the outcome. Unfallchirurg 1991; 94(6): 325-30 (Article in German)

Bryant C, Harley J. Re: Parkinson's disease patients who fracture their neck of femur: a review of outcome data. Injury 2007; 38(10): 1222.

Butt FF, Hussain AS, Khan AM, Sultan MJ. Implants for extracapsular neck of femur fracture Dynamic hip screw versus intramedullary nailing. J Ayub Med Coll Abbottabad 2017; 29(4): 697-701.

Florschutz AV, Langford JR, Haidukewych GJ, Koval KJ. Femoral neck fractures: current management. J Orthop Trauma 2015; 29(3): 1219.

Gullberg B, Johnell O, Kanis JA. World-wide projections for hip fracture. Osteoporosis Int; 1997: 407-13.

Langford J, Strauss E. Femoral Neck Fractures. Fix or Replace? Techniques in Orthopedics 2008; 23(3): 208-12.

Lee YS, Chen SH, Tsuang YH, Huang HL, Lo TY, Huang CR. Internal fixation of undisplaced femoral neck fractures in the elderly: A retrospective comparison of fixation methods. J Trauma 2008; 64: 155-62.

Li M, Cole PA. Anatomical considerations in adult femoral neck fractures: how anatomy influences the treatment issues? Injury 2015; 46(3): 453-8.

Lin PP, Kang HG, Kim YI, Kim JH, Kim HS. Minimally invasive surgery for femoral neck fractures using bone cement infusible hollowperforated screw in high-risk patients with advanced cancer. Surg Oncol. 2015; 24(3): 22631.

Malik AA, Kell P, Khan WS, Ihsan KM, Dunkow PJ. Surgical management of fractured neck of femur. Perioper Pract 2009; 19(3): 100-4.

Martyn J. Fractures of the hip. Surgery 2003; 21: $221-4$.

Ong BC, Maurer SG, Aharonoff GB, Zuckerman JD, Koval KJ. Unipolar versus bipolar hemiarthroplasty: Functional outcome after femoral neck fracture at a minimum of thirty-six months of follow-up. J Orthop Trauma 2002; 16: 317-22.

Parker MJ. The management of intracapsular fractures of the proximal femur. J Bone Joint Surg (Br) 2000; 82-B: 937- 41. 
Patterson JT, Tangtiphaiboontana J, Pandya NK. Management of Pediatric Femoral Neck Fracture. J Am Acad Orthop Surg. 2018: 26(12): 411-9.

Thuan V Ly, Swiontkowski MF. Management of femoral neck fractures in young adults. Indian $\mathbf{J}$ Orthop 2008; 42(1): 3-12.

Weil NL, van Embden D, Hoogendoorn JM. Radiographic fracture features predicting failure of internal fixation of displaced femoral neck fractures. Eur J Trauma Emerg Surg 2015; 41(5): 501-7.

Yamauchi K, Naofumi M, Sumida H, Fukuta S, Hori $\mathrm{H}$. Comparison of morphological features in the femur between femoral neck fractures and femoral intertrochanteric fractures. Surg Radiol Anat 2016; 38(7): 775-80.

Zlowodzki M, Ayeni O, Petrisor BA, Bhandari M. Femoral neck shortening after fracture fixation with multiple cancellous screws: incidence and effect on function. J Trauma 2008: 64(1): 163-9. 